\title{
Image Preprocessing and Filtering Effect on the Estimate of Myocardial Radiomic Features From T1 and T2 Mapping in Hypertrophic Cardiomyopathy
}

\author{
Daniela Marfisi \\ Pisa University Hospital "Azienda Ospedaliero-Universitaria Pisana" \\ Carlo Tessa \\ Azienda USL Toscana Nord Ovest, Apuane Hospital \\ Chiara Marzi \\ Institute of Applied Physics "Nello Carrara" - Italian National Research Council \\ Jacopo Del Meglio \\ Azienda USL Toscana Nord Ovest, Versilia Hospital \\ Stefania Linsalata \\ Pisa University Hospital "Azienda Ospedaliero-Universitaria Pisana" \\ Rita Borgheresi \\ Pisa University Hospital "Azienda Ospedaliero-Universitaria Pisana" \\ Alessio Lilli \\ Azienda USL Toscana Nord Ovest, Versilia Hospital \\ Riccardo Lazzarini \\ Azienda USL Toscana Nord Ovest, Versilia Hospital \\ Luca Salvatori \\ Azienda USL Toscana Nord Ovest, Versilia Hospital \\ Claudio Vignali \\ Azienda USL Toscana Nord Ovest, Versilia Hospital

\section{Andrea Barucci} \\ Institute of Applied Physics "Nello Carrara" - Italian National Research Council \\ Mario Mascalchi \\ University of Florence \\ Giancarlo Casolo \\ Azienda USL Toscana Nord Ovest, Versilia Hospital \\ Stefano Diciotti \\ University of Bologna \\ Antonio Claudio Traino \\ Pisa University Hospital "Azienda Ospedaliero-Universitaria Pisana" \\ Marco Giannelli (D m.giannelli@ao-pisa.toscana.it) \\ Pisa University Hospital "Azienda Ospedaliero-Universitaria Pisana"
}

\section{Research Article}

Keywords: Radiomics, cardiac magnetic resonance (CMR) imaging, CMR T1 and T2 mapping, myocardial diseases

Posted Date: August 12th, 2021

DOI: https://doi.org/10.21203/rs.3.rs-798103/v1

License: (c) (i) This work is licensed under a Creative Commons Attribution 4.0 International License. Read Full License 


\section{Abstract}

Radiomics is emerging as a promising and useful tool in cardiac magnetic resonance (CMR) imaging applications. Accordingly, the purpose of this study was to investigate, for the first time, the effect of image preprocessing and filtering on radiomic features estimation from quantitative CMR T1 and T2 mapping. Specifically, T1 and T2 maps of 26 patients with hypertrophic cardiomyopathy (HCM) were used to estimate 98 radiomic features for 7 different resampling voxel sizes (at fixed bin width), 9 different bin widths (at fixed resampling voxel size), and 7 different spatial filters (at fixed resampling voxel size/bin width). While we found a remarkable dependence of myocardial radiomic features from T1 and T2 mapping on image filters, many radiomic features showed a limited sensitivity to resampling voxel size/bin width, in terms of intraclass correlation coefficient ( $>0.75)$ and coefficient of variation $(<30 \%)$. The estimate of several textural radiomic features showed a linear significant $(p<0.05)$ correlation with resampling voxel size/bin width. Overall, radiomic features from T2 maps have proven to be less sensitive to image preprocessing than those from T1 maps, especially when varying bin width. Our results might corroborate the potential of radiomics from T1/T2 mapping in HCM and hopefully in other myocardial diseases.

\section{Introduction}

Hypertrophic cardiomyopathy (HCM) is a genetically determined disease that affects about 1:500 people in the general adult population ${ }^{1}$. It is characterized by left ventricular (LV) hypertrophy, myofibrillar disarray, and myocardial fibrosis. Cardiac magnetic resonance (CMR) imaging has an important role in diagnosis, risk stratification, and treatment planning in HCM and, according to the European Society of Cardiology guidelines, it should be performed, at least as an initial evaluation, for all HCM patients ${ }^{2}$.

Actually, CMR imaging is considered the gold standard for the evaluation of ventricular morphology and function, as well as for the assessment of ventricular wall thickness ${ }^{3,4}$. Furthermore, late gadolinium enhancement (LGE) imaging enables the identification of focal myocardial fibrosis, which has been demonstrated to have a negative prognostic value in HCM patients ${ }^{3,4}$. More recently, cardiac T1 and T2 mapping techniques have been employed to obtain a more quantitative evaluation of myocardial tissue characteristics. Previous studies have consistently found that T1 and T2 values, as well as extracellular volume (ECV) values obtained from pre- and post-contrast T1 maps, are increased in HCM patients and have a potential prognostic role ${ }^{5-11}$. Actually, both native (i.e., without contrast administration) T1 and ECV values are considered markers of myocardial fibrosis ${ }^{12}$. On the other hand, T2 mapping is currently considered the gold standard for the evaluation of myocardial edema. Increased T2 values, probably related to low grade myocardial inflammation and dilated lymphatic channels ${ }^{13,14}$, have been demonstrated in HCM patients and have been proven to be related to an increased arrhythmic risk ${ }^{15,16}$, as well as to more severe myocardial injury ${ }^{17}$. However, the usefulness of these mapping techniques is limited by a partial overlap of $\mathrm{T} 1$ and $\mathrm{T} 2$ values between HCM patients and healthy controls, as well as between HCM patients and patients with other myocardial diseases ${ }^{18-21}$.

Radiomics is a novel tool that involves the extraction of a large number of quantitative morphological and textural characteristics (i.e., radiomic features) from digital medical images ${ }^{22}$. The underlying idea is that medical images are data containing objective and quantitative information, which is not obtainable from qualitative visual inspection as usually performed in routine clinical practice. In addition to other available data from demographics, pathology, blood biomarkers, and genomics, radiomic features can be used for diagnostic, prognostic or predictive purposes exploiting statistical or machine learning $(\mathrm{ML})$ methods ${ }^{22-24}$

While radiomic analysis has been mainly applied in the field of oncology, there is a growing interest in improving the diagnostic accuracy and prognostic value of CMR imaging by exploiting radiomic techniques ${ }^{25-30}$. Recently, radiomics has aroused as a useful tool for unveiling myocardial tissue characteristics in HCM patients ${ }^{18-21,31-36}$. In this regard, Baeßler et al. ${ }^{31}$ have reported significant differences in texture features obtained from T1weighted images of HCM patients and healthy subjects, identifying a single feature (i.e., gray level non uniformity from the gray level run length matrix) as the best discriminator. Schofield et al. ${ }^{32}$ have found that texture analysis of cine CMR images was able to differentiate patients with HCM, cardiac amyloid, aortic stenosis, and healthy subjects. Moreover, some studies have focused on the prognostic role of radiomic analysis of LGE images in HCM finding that texture features may be significantly related to the arrhythmic risk ${ }^{33}$ and adverse clinical outcome ${ }^{34}$.

Given their inherent quantitative nature, T1 and T2 maps might be particularly suitable for radiomic analysis. So far, few studies have applied radiomic analysis to $\mathrm{T} 1$ maps in patients with $\mathrm{HCM}^{19-21,36}$. In two studies of the same group, radiomic analysis of native $\mathrm{T} 1$ maps has allowed discriminating between hypertensive heart disease (HHD) and $\mathrm{HCM}^{19}$, as well as between LGE + and LGE- patients, identifying a subgroup of $\mathrm{HCM}$ patients without scar in whom gadolinium administration could be avoided ${ }^{36}$. Moreover, Wang et al. ${ }^{20}$ have found that radiomic analysis of native T1 maps can discriminate HCM patients with different mutations of sarcomere-related genes. Shi et al. ${ }^{21}$ have shown that texture analysis of native T1 and ECV maps could distinguish between patients (HCM and HHD) and healthy subjects, as well as between HCM and HHD, with diagnostic accuracies comparable with strain parameters.

Despite the increasing interest in radiomics of CMR imaging, its proper application deserves some caution and a preliminary assessment of possible radiomic features dependence on various factors. Indeed, each step of the radiomic workflow (i.e., image acquisition and reconstruction, image segmentation, image preprocessing, image filtering, and feature extraction) could influence features estimation, thus potentially affecting their discriminative or predictive power ${ }^{37,38}$. Several previous studies have assessed the sensitivity of radiomic features estimation to various elements in computed tomography (CT) ${ }^{39-41}$ and nuclear medicine (NM) ${ }^{42,43}$ imaging, as well as in magnetic resonance imaging (MRI), for various applications ${ }^{44-55}$. On the other hand, only three studies have assessed some of these issues for CMR imaging ${ }^{56-58}$. In particular, Jang et al. ${ }^{56}$ have investigated both test- 
retest repeatability, as well as inter- and intra-observer reproducibility, in phantoms, healthy participants, and patients who were referred for clinical CMR imaging. They have observed different repeatability patterns among acquisition sequences in in vivo test-retest studies. Moreover, in an inter- and intraobserver reproducibility analysis, $32 \%-47 \%$ and $61 \%-73 \%$ out of 1023 features, respectively, were identified as reproducible, showing intraclass correlation coefficient (ICC) $\geq 0.8$. Across all analyses, first order and gray level co-occurrence matrix were the most frequently identified reproducible radiomic feature classes. Another CMR imaging study by Jang et al. ${ }^{57}$ has evaluated, in a group of healthy participants and in a heterogeneous group of patients, the sensitivity of radiomic features estimate to changes in acquisition sequence parameters including flip angle, in-plane spatial resolution, slice thickness, and parallel imaging technique. As a whole, they have found that approximately $60 \%$ of the 4007 considered radiomic features were robust to changes in any acquisition parameters (i.e., a standardized Cohen's mean difference $<0.2$ ), with qualitative acquisition sequences (i.e., cine balanced steady-state free-precession, T1-weighted spoiled gradient-echo, T2-weighted turbo spin-echo) and quantitative T1/T2 mapping most sensitive to changes in in-plane spatial resolution and flip angle, respectively. Alis et al. ${ }^{58}$ have evaluated the inter-observer reproducibility of radiomic features, as well as the influence of cardiac phases (i.e., systole, diastole or a 4D stack of cine images covering the cardiac cycle) on radiomic features estimation, from non-enhanced cine CMR images of healthy participants. For the inter-observer analysis, they have found that the number of radiomic features with ICC $>0.8 / 0.85 / 0.9$ changed with different phases of the cardiac cycle, and that texture features belonging to the gray level size zone matrix showed the worst performance. Moreover, approximately $55 \%$ out of 352 radiomic features showed a variability (in terms of coefficient of variation) $>20 \%$ through the cardiac cycle, with gray level dependence matrix and gray level run length matrix classes demonstrating less variability compared with other feature classes.

Image preprocessing and filtering are two steps of the radiomic workflow that may be performed before the radiomic features extraction from the acquired image data ${ }^{59,60}$. In particular, image interpolation at the same voxel size is a common and recommended practice (especially in retrospective studies) to reduce any heterogeneity in acquisition voxel size, while image discretization is required to make texture feature estimation computationally less burdensome ${ }^{42,59}$. Moreover, the application of spatial filters before radiomic features estimation could allow uncovering further tissue characteristics. Notably, preprocessing and spatial filter actually alter acquired image data. This can result in some sensitivity of radiomic features to the applied image preprocessing and filter. Nonetheless, so far, no work has specifically assessed the impact of both image preprocessing and image filtering on the estimate of radiomic features from CMR imaging. Therefore, this work aimed to comprehensively assess, for the first time, the effect of image preprocessing-in terms of resampling voxel size and discretization-and image filtering on radiomic features estimated from CMR T1 and T2 mapping in a group of HCM patients.

\section{Methods \\ Subjects}

We retrospectively identified 26 subjects (12 females, 14 males), referred for clinical CMR imaging between November 2013 and July 2020 for known or suspected HCM, for whom a comprehensive MRI study including both T1 and T2 mapping sequences was performed. The HCM diagnoses followed the latest European Society of Cardiology guidelines and were based on the presence of LV wall thickness $\geq 15 \mathrm{~mm}$ in one or more myocardial segments, not explained solely by loading conditions ${ }^{2}$. Clinical characteristics of the patient group are detailed in Table 1 . The study was approved by the local ethics committee of the Azienda USL Toscana Nord Ovest (Pisa, Italy). Written informed consent was obtained from all subjects, and all experiments were performed in accordance with relevant guidelines and regulations.

Table 1

Clinical characteristics and CMR parameters (mean \pm standard deviation) of 26 patients with HCM.

\begin{tabular}{|ll|}
\hline Parameters \\
\hline Age (years) & $66 \pm 11$ \\
\hline LV ED volume $\left(\mathrm{ml} / \mathrm{m}^{2}\right)$ & $74 \pm 15$ \\
\hline LV ES volume $\left(\mathrm{ml} / \mathrm{m}^{2}\right)$ & $23 \pm 14$ \\
\hline LV stroke volume $\left(\mathrm{ml} / \mathrm{m}^{2}\right)$ & $51 \pm 13$ \\
\hline LV ejection fraction $(\%)$ & $70 \pm 15$ \\
\hline Mass $\left(\mathrm{g} / \mathrm{m}^{2}\right)$ & $96 \pm 21$ \\
\hline RV ED volume $\left(\mathrm{ml} / \mathrm{m}^{2}\right)$ & $61 \pm 13$ \\
\hline RV ES volume $\left(\mathrm{ml} / \mathrm{m}^{2}\right)$ & $23 \pm 6$ \\
\hline RV stroke volume $\left(\mathrm{ml}^{2} / \mathrm{m}^{2}\right)$ & $38 \pm 9$ \\
\hline RV ejection fraction $(\%)$ & $63 \pm 6$ \\
\hline Thickness (mm) & $19 \pm 3$ \\
\hline
\end{tabular}

Page $3 / 18$ 


\section{CMR imaging}

All CMR imaging examinations were performed using a 1.5 T MRI scanner system (MAGNETOM Avanto, Siemens Healthcare, Erlangen, Germany) equipped with $45 \mathrm{mT} / \mathrm{m}$ gradients strength and a 12-channel surface phased array coils.

Cine images were performed in the 2-and 4-chamber view planes (3 slices each) and in short-axis view (8-14 slices encompassing the entire LV), using a TrueFISP sequence $(\mathrm{TR}=2.5 \mathrm{~ms}, \mathrm{TE}=1.2 \mathrm{~ms}$, slice thickness $=8 \mathrm{~mm})$.

Both T1 and T2 maps were obtained in the short axis view (a single slice located where myocardial thickness, evaluated with cine images, was maximum). T1 mapping was performed utilizing a modified look-locker inversion recovery (MOLLI) pulse sequence with a 3-3-5 acquisition scheme ${ }^{61}$ Pulse sequence parameters were as follows: TE $/ \mathrm{TR}=1.14 / 2.5 \mathrm{~ms}$, flip angle $=35^{\circ}$, matrix size $=124 \times 192$, typical in-plane resolution ranged from 1.8 $\mathrm{mm} \times 1.8 \mathrm{~mm}$ to $2.2 \mathrm{~mm} \times 2.2 \mathrm{~mm}$, typical field of view $=380 \mathrm{~mm} \times 273 \mathrm{~mm}$, slice thickness $=8 \mathrm{~mm}$. T2 maps were obtained utilizing a T2-prepared TrueFISP sequence ${ }^{62}$ with the following parameters: T2 preparation time $=0 / 24 / 55 \mathrm{~ms}, \mathrm{TR}=4 \times R-R$, flip angle $=70^{\circ}$, matrix size $=126 \times 192$, typical inplane resolution ranged from $1.8 \mathrm{~mm} \times 1.8 \mathrm{~mm}$ to $2.2 \mathrm{~mm} \times 2.2 \mathrm{~mm}$, typical field of view $=380 \mathrm{~mm} \times 276 \mathrm{~mm}$, slice thickness $=6 \mathrm{~mm}$.

Then, 10-15 minutes after intravenous administration of $0.2 \mathrm{mmol} / \mathrm{kg}$ of gadolinium DTPA (Magnevist, Schering), gadoteric acid (Dotarem, Guerbet) or gadoteridol (Prohance, Bracco), LGE images were acquired in 2- and 4-chamber view of the LV (3 slices each), as well as in the short-axis view (8-14 slices encompassing the entire LV) using a 2D phase-sensitive inversion recovery (PSIR) sequence $(T R=700 \mathrm{~ms}, T E=1.09 \mathrm{~ms}$, slice thickness $=8 \mathrm{~mm}$, inversion time $=200-300 \mathrm{~ms}$ ).

\section{Image preprocessing and filtering of T1 and T2 maps}

For each subject, a region of interest (ROI) covering the entire myocardium was manually segmented by a single expert radiologist in cardiac MRI, with 15 years of experience, using 3D Slicer (Version 4.11.2) ${ }^{63,64}$. ROIs were delineated on T1 and T2 maps separately, avoiding voxels with potential partial volume effect.

Given that T1 and T2 maps allowed acquiring only a single slice, resampling voxel size was performed through 2D interpolation by using the B-spline interpolation algorithm (with the origins of interpolation and original image grids aligned together ${ }^{59}$ ). The original in-plane spatial resolution ranged from $1.77 \mathrm{~mm} \times 1.77 \mathrm{~mm}$ to $2.34 \mathrm{~mm} \times 2.34 \mathrm{~mm}$ across patients for both T1 and T2 maps. Therefore, T1 and T2 maps were resampled to an in-plane isotropic spatial resolution of $1.8 \mathrm{~mm}, 1.9 \mathrm{~mm}, 2.0 \mathrm{~mm}, 2.1 \mathrm{~mm}, 2.2 \mathrm{~mm}, 2.3 \mathrm{~mm}$, and $2.4 \mathrm{~mm}$. Resampling voxel size was performed for different bin widths (see below).

T1 and T2 maps discretization was carried out following a fixed bin width approach, as recommended by the Image Biomarker Standardisation Initiative (IBSI) when dealing with quantitative data ${ }^{59}$. In particular, for each resampling voxel size (i.e., 1.8, 1.9, 2, 2.1, 2.2, 2.3, and 2.4 mm in-plane isotropic spatial resolution), bin width values of $3.60 \mathrm{~ms}, 3.95 \mathrm{~ms}, 4.30 \mathrm{~ms}, 4.65 \mathrm{~ms}, 5.00 \mathrm{~ms}, 5.35 \mathrm{~ms}, 5.70 \mathrm{~ms}, 6.05 \mathrm{~ms}$, and $6.40 \mathrm{~ms}$ were employed for T1 maps, while bin width values of $0.49 \mathrm{~ms}, 0.50 \mathrm{~ms}, 0.51 \mathrm{~ms}, 0.52 \mathrm{~ms}, 0.53 \mathrm{~ms}, 0.54 \mathrm{~ms}, 0.55 \mathrm{~ms}, 0.56 \mathrm{~ms}$, and 0.57 ms were employed for T2 maps. Indeed, these bin width values yielded median (across subjects) numbers of quantization levels between 30 and 13043,65,66.

Different spatial filters were applied on T1 and T2 maps: 2D wavelets (Daubechies 3) yielding four filtered maps (i.e., wavelet-LH, -HL, -HH, -LL, where L/H refers to the combination of low-/high-pass filters applied in the horizontal and vertical direction), gradient magnitude of the map (i.e., gradient filter), the square of the map values (i.e., square filter), and the square root of the absolute map value (i.e., square-root filter). Specifically, in order to restrict the large number of possible combinations, image filtering was performed at fixed resampling in-plane isotropic spatial resolution of $2.1 \mathrm{~mm}$, and at fixed bin width of $6 \mathrm{~ms}$ and $0.56 \mathrm{~ms}$ for T1 and T2 maps, respectively, yielding median (across subjects) numbers of quantization levels between 30 and $130^{43,65,66}$.

All preprocessing steps, spatial filters application, and subsequent radiomic features estimation were carried out by using the open source PyRadiomics library ${ }^{67}$ (Version 3.0.1) with Python (Version 3.7.3).

\section{Radiomic features estimation}

Given that the used acquisition sequences allowed obtaining T1 and T2 maps on a single slice, the 2D versions of radiomic features were considered. For each ROI and preprocessing combination, in terms of resampling voxel size and bin width, a total of 98 features were obtained: 9 2D-shape features, 16 first order features (14 intensity-based statistical features and 2 intensity histogram features, namely Entropy and Uniformity), and 73 second order features (i.e., textural features) from gray level co-occurrence matrix (GLCM, 22 features), gray level run length matrix (GLRLM, 16 features), gray level size zone matrix (GLSZM, 16 features), gray level dependence matrix (GLDM, 14 features, with coarseness parameter $a=0$ ), and neighborhood gray tone difference matrix (NGTDM, 5 features). Second order features estimation was performed according to the Chebyshev norm with a distance of 1 pixel. GLCM and GLRLM features were computed from each 2D directional matrix (i.e., at $0^{\circ}, 45^{\circ}, 90^{\circ}$, and $135^{\circ}$ ) and averaged over $2 \mathrm{D}$ directions.

For each ROI and spatial filter applied on T1 and T2 maps, a total of 89 features were estimated, i.e. all the above features but the shape features. Indeed, given that shape features are usually estimated regardless of the applied image filter, they were not included in our analysis.

All radiomic features were computed in accordance with the definitions provided by the IBSI, with shape features computed in 2D instead of the proposed 3D version. It is worth noting that the first order feature of Kurtosis calculated by PyRadiomics was in accordance with the IBSI except for an offset value 
(i.e., 3).

\section{Statistical analysis}

In this study, three different effects on radiomic features estimation were assessed for T1 and T2 maps singularly:

1. For each bin width, the effect of using different resampling voxel sizes;

2. For each voxel size, the effect of using different bin widths;

3. At fixed resampling voxel size and bin width, the effect of using different spatial filters.

For each effect of interest, any variability in radiomic features estimate was assessed through ICC analysis ${ }^{68,69}$. In particular, the two-way mixed effects model, with single rater and absolute agreement, was selected. Accordingly, the ICC coefficient was calculated as:

$$
I C C=\frac{M S_{R}-M S_{E}}{M S_{R}+(k-1) M S_{E}+\frac{k}{n}\left(M S_{C}-M S_{E}\right)}
$$

where $\mathrm{MS}_{\mathrm{R}}=$ mean square for rows (i.e., between subjects), $\mathrm{MS}_{\mathrm{E}}=$ mean square for error, $\mathrm{MS}_{\mathrm{C}}=$ mean square for columns (i.e., between measurements), $\mathrm{n}=$ number of subjects and $\mathrm{k}=$ number of raters, with ICC matrices realized considering each resampling voxel size, quantization bin width or image filter as a rater. ICC values range between 0 (maximum variability) and 1 (minimum variability) and express the variability of radiomic features estimate associated with the effect of interest with respect to the variance between subjects (i.e., a relative variability). Radiomic features were stratified based on their degree of relative variability ${ }^{37,52}$ : high $(\mathrm{ICC} \leq 0.5)$, considerable $(0.5<\mathrm{ICC} \leq 0.75)$, moderate $(0.75<\mathrm{ICC} \leq 0.9)$, and low $(0.9<\mathrm{ICC} \leq 1)$ relative variability.

In order to characterize in greater detail and absolutely the variability of radiomic feature estimation, an additional analysis of the coefficient of variation (CV) was performed. In particular, CV were computed as the percentage ratio between standard deviation and absolute mean values of features estimate obtained by varying one element of interest (resampling voxel size, bin width or spatial filter) while fixing the others. Accordingly, this yielded $26 \times 9 \mathrm{CVs}$ for effect A (i.e., 26 subjects and 9 fixed bin widths), $26 \times 7$ CVs for effect B (i.e., 26 subjects and 7 fixed resampling voxel size), and $26 \mathrm{CVs}$ for effect $\mathrm{C}$ (i.e., 26 subjects with fixed resampling voxel size and bin width).

Any linear correlation between radiomic features estimate and resampling voxel size or bin width was assessed by a repeated measures correlation analysis, namely $\mathrm{rmcorr}^{70}$. This technique accounts for non-independence among observations (i.e., repeated measures of the same radiomic features of the same subject by varying one preprocessing element) using analysis of covariance (ANCOVA) to statistically adjust for the variability between subjects. A p-value < 0.05, adjusted for multiple comparisons using Bonferroni correction, was considered significant.

Statistical analysis was carried out by using R (Version 3.6.2) software package in the RStudio (Version 1.2.5033) environment ${ }^{71}$.

\section{Results}

ICC results for the effects of interest A, B, and C are reported in Figs. 1, 2, and 3, respectively, for both T1 and T2 maps. ICC values were $>0.9$ for radiomic features belonging to the shape class. In general, for effects $A$ and $B$, first and second order features estimated from T2 maps showed a lower relative variability than those estimated from T1 maps, especially for effect B where ICC values were always in moderate or low ranges (with few exceptions for some resampling voxel size values, i.e., GLSZM-LargeAreaLowGrayLevelEmphasis, GLSZM-SmallAreaLowGrayLevelEmphasis, and GLDMLargeDependenceLowGrayLevelEmphasis). For both $\mathrm{T} 1$ and $\mathrm{T} 2$ maps, effect $\mathrm{C}$ resulted in higher relative variability (ICC $\leq 0.5)$ of radiomic features estimate as compared to effect $A$ and $B$.

For effects A and B, Table 2 summarizes the median (across subjects and bin widths/voxel sizes for effect A/B) CV values of radiomic features estimated from T1 and T2 maps. The CV was less than 40\% for both effects as well as for T1 and T2 (with few exceptions for effect B assessed on T1 maps, i.e., GLCM-ClusterShade, GLCM-ClusterProminence, NGTDM-Complexity, and GLDM-SmallDependenceHighGrayLevelEmphasis). Specifically, for effect B assessed on T2 maps, this absolute variability was $<20 \%$. Moreover, Figs. $4 / 5$ a and Figs. $5 \mathrm{~b} / 5 \mathrm{c}$ show radiomic features with both median (across subjects and bin widths/voxel sizes for effect A/B) CV > 15\% and ICC $>0.75$ for effect A/B assessed on T1 and T2 maps, respectively. For effect C, median (across subjects) CV values of radiomic features estimated from T1 and T2 maps are summarized in Table 3, showing that the absolute variability associated with this effect was markedly greater than that associated with effects $A$ and $B$.

Table 2. Median CV (\%) of radiomic features estimate for effect A and B for both T1 and T2 maps. The median CV was calculated across subjects and bin widths/voxel sizes for effect A/B. 


\begin{tabular}{|c|c|c|c|c|c|c|c|c|c|}
\hline Shape & $\begin{array}{l}\text { A } \\
\text { (T1) }\end{array}$ & $\begin{array}{l}\text { B } \\
(\mathrm{T} 1)\end{array}$ & $\begin{array}{l}\text { A } \\
\text { (T2) }\end{array}$ & $\begin{array}{l}\text { B } \\
\text { (T2) }\end{array}$ & GLRLM & $\begin{array}{l}\text { A } \\
\text { (T1) }\end{array}$ & $\begin{array}{l}\text { B } \\
\text { (T1) }\end{array}$ & $\begin{array}{l}\text { A } \\
\text { (T2) }\end{array}$ & $\begin{array}{l}\text { B } \\
\text { (T2) }\end{array}$ \\
\hline Elongation & 1.51 & 0.00 & 1.65 & 0.00 & GrayLevelNonUniformity & 17,23 & 14,81 & 18,14 & 4,28 \\
\hline MajorAxisLength & 0.99 & 0.00 & 0.82 & 0.00 & GrayLevelNonUniformityNormalized & 3,32 & 16,00 & 2,77 & 4,55 \\
\hline MaximumDiameter & 0.94 & 0.00 & 0.84 & 0.00 & GrayLevelVariance & 7,31 & 37,55 & 7,20 & 9,70 \\
\hline MeshSurface & 2.67 & 0.00 & 2.35 & 0.00 & HighGrayLevelRunEmphasis & 17,16 & 36,92 & 10,12 & 9,55 \\
\hline MinorAxisLength & 1.03 & 0.00 & 0.90 & 0.00 & LongRunEmphasis & 2,54 & 3,39 & 1,99 & 1,27 \\
\hline Perimeter & 1.27 & 0.00 & 0.96 & 0.00 & LongRunHighGrayLevelEmphasis & 16,95 & 33,68 & 10,52 & 8,88 \\
\hline PerimeterSurfaceRatio & 2.55 & 0.00 & 2.39 & 0.00 & LongRunLowGrayLevelEmphasis & 26,94 & 17,67 & 25,70 & 8,58 \\
\hline PixelSurface & 2.66 & 0.00 & 2.36 & 0.00 & LowGrayLevelRunEmphasis & 23,29 & 15,45 & 22,44 & 7,68 \\
\hline \multirow[t]{2}{*}{ Sphericity } & 1.71 & 0.00 & 1.51 & 0.00 & RunEntropy & 1,31 & 3,49 & 1,16 & 1,04 \\
\hline & & & & & RunLengthNonUniformity & 17,61 & 3,25 & 18,48 & 1,24 \\
\hline first order & $\begin{array}{l}\text { A } \\
\text { (T1) }\end{array}$ & $\begin{array}{l}\text { B } \\
(\mathrm{T} 1)\end{array}$ & $\begin{array}{l}\text { A } \\
\text { (T2) }\end{array}$ & $\begin{array}{l}\text { B } \\
\text { (T2) }\end{array}$ & RunLengthNonUniformityNormalized & 1,53 & 2,12 & 1,28 & 0,83 \\
\hline Mean & 0.17 & 0.00 & 0.23 & 0.00 & RunPercentage & 0.83 & 1.13 & 0.68 & 0.42 \\
\hline Variance & 7.07 & 0.00 & 7.10 & 0.00 & RunVariance & 17.10 & 22.36 & 14.31 & 8.65 \\
\hline Skewness & 23.38 & 0.00 & 36.34 & 0.00 & ShortRunEmphasis & 0.61 & 0.85 & 0.51 & 0.33 \\
\hline Kurtosis & 6.25 & 0.00 & 15.42 & 0.00 & ShortRunHighGrayLevelEmphasis & 17.31 & 37.67 & 10.18 & 9.74 \\
\hline Median & 0.14 & 0.00 & 0.19 & 0.00 & ShortRunLowGrayLevelEmphasis & 22.61 & 14.62 & 22.31 & 7.55 \\
\hline Minimum & 2.18 & 0.00 & 1.28 & 0.00 & & & & & \\
\hline 10Percentile & 0.33 & 0.00 & 0.26 & 0.00 & GLSZM & $\begin{array}{l}\text { A } \\
\text { (T1) }\end{array}$ & $\begin{array}{l}\text { B } \\
(\mathrm{T} 1)\end{array}$ & $\begin{array}{l}\text { A } \\
\text { (T2) }\end{array}$ & $\begin{array}{l}\text { B } \\
\text { (T2) }\end{array}$ \\
\hline 90Percentile & 0.26 & 0.00 & 0.57 & 0.00 & GrayLevelNonUniformity & 15.14 & 11.14 & 17.05 & 3.74 \\
\hline Maximum & 1.56 & 0.00 & 4.08 & 0.00 & GrayLevelNonUniformityNormalized & 3.98 & 15.26 & 3.20 & 4.56 \\
\hline InterquartileRange & 3.39 & 0.00 & 3.32 & 0.00 & GrayLevelVariance & 7.64 & 36.63 & 7.60 & 9.51 \\
\hline MeanAbsoluteDeviation & 3.16 & 0.00 & 2.50 & 0.00 & HighGrayLevelZoneEmphasis & 16.89 & 36.87 & 9.77 & 9.47 \\
\hline RobustMeanAbsoluteDeviation & 2.61 & 0.00 & 2.45 & 0.00 & LargeAreaEmphasis & 10.03 & 14.02 & 8.17 & 5.17 \\
\hline Energy & 19.05 & 0.00 & 19.44 & 0.00 & LargeAreaHighGrayLevelEmphasis & 21.57 & 24.31 & 13.95 & 7.79 \\
\hline RootMeanSquared & 0.17 & 0.00 & 0.24 & 0.00 & LargeAreaLowGrayLevelEmphasis & 29.79 & 26.74 & 29.44 & 11.17 \\
\hline Entropy & 0.93 & 4.70 & 0.88 & 1.36 & LowGrayLevelZoneEmphasis & 21.64 & 15.94 & 20.50 & 6.43 \\
\hline \multirow[t]{2}{*}{ Uniformity } & 3.22 & 16.23 & 2.78 & 4.65 & SizeZoneNonUniformity & 14.60 & 10.78 & 16.61 & 4.46 \\
\hline & & & & & SizeZoneNonUniformityNormalized & 4.86 & 6.38 & 4.19 & 2.82 \\
\hline GLCM & $\begin{array}{l}\text { A } \\
\text { (T1) }\end{array}$ & $\begin{array}{l}\text { B } \\
\text { (T1) }\end{array}$ & $\begin{array}{l}\text { A } \\
\text { (T2) }\end{array}$ & $\begin{array}{l}\text { B } \\
\text { (T2) }\end{array}$ & SmallAreaEmphasis & 2.19 & 2.93 & 1.90 & 1.26 \\
\hline MaximumProbability & 12.90 & 18.94 & 11.52 & 8.92 & SmallAreaHighGrayLevelEmphasis & 17.62 & 39.43 & 10.35 & 10.19 \\
\hline JointAverage & 9.00 & 18.28 & 6.05 & 4.80 & SmallAreaLowGrayLevelEmphasis & 22.68 & 14.50 & 22.84 & 7.56 \\
\hline SumSquares & 6.43 & 37.89 & 5.43 & 9.78 & ZoneEntropy & 1.99 & 2.24 & 1.74 & 0.88 \\
\hline JointEntropy & 2.01 & 2.48 & 1.78 & 0.84 & ZonePercentage & 3.25 & 4.48 & 2.67 & 1.75 \\
\hline DifferenceAverage & 7.19 & 18.81 & 6.32 & 4.90 & ZoneVariance & 25.96 & 31.92 & 22.03 & 13.92 \\
\hline DifferenceVariance & 15.11 & 37.67 & 15.53 & 9.73 & & & & & \\
\hline DifferenceEntropy & 2.12 & 6.35 & 2.09 & 1.72 & GLDM & $\begin{array}{l}\text { A } \\
\text { (T1) }\end{array}$ & $\begin{array}{l}\text { B } \\
\text { (T1) }\end{array}$ & $\begin{array}{l}\text { A } \\
\text { (T2) }\end{array}$ & $\begin{array}{l}\text { B } \\
\text { (T2) }\end{array}$ \\
\hline SumEntropy & 1.33 & 3.70 & 1.13 & 1.12 & DependenceEntropy & 2.20 & 1.81 & 1.92 & 0.82 \\
\hline JointEnergy & 9.57 & 16.96 & 8.26 & 5.66 & DependenceNonUniformity & 14.85 & 9.97 & 16.66 & 3.93 \\
\hline Contrast & 14.07 & 37.79 & 13.24 & 9.77 & DependenceNonUniformityNormalized & 6.99 & 9.97 & 5.74 & 3.93 \\
\hline
\end{tabular}




\begin{tabular}{|c|c|c|c|c|c|c|c|c|c|}
\hline Id & 4.44 & 10.53 & 3.90 & 2.92 & DependenceVariance & 19.39 & 21.59 & 15.42 & 9.81 \\
\hline Idn & 0.76 & 0.05 & 0.97 & 0.06 & GrayLevelNonUniformity & 18.14 & 16.23 & 18.64 & 4.65 \\
\hline Idm & 6.67 & 15.30 & 5.89 & 4.28 & GrayLevelVariance & 7.16 & 37.90 & 7.07 & 9.78 \\
\hline Idmn & 0.26 & 0.02 & 0.32 & 0.02 & HighGrayLevelEmphasis & 17.22 & 36.96 & 10.38 & 9.60 \\
\hline InverseVariance & 6.69 & 15.05 & 6.45 & 4.77 & LargeDependenceEmphasis & 10.01 & 13.61 & 8.18 & 5.31 \\
\hline Correlation & 5.44 & 0.15 & 7.95 & 0.25 & LargeDependenceHighGrayLevelEmphasis & 22.07 & 24.58 & 14.42 & 7.93 \\
\hline Autocorrelation & 17.22 & 36.95 & 11.00 & 9.62 & LargeDependenceLowGrayLevelEmphasis & 35.67 & 28.91 & 33.90 & 12.69 \\
\hline ClusterTendency & 7.43 & 37.89 & 6.33 & 9.79 & LowGrayLevelEmphasis & 24.40 & 15.39 & 23.19 & 7.65 \\
\hline ClusterShade & 28.91 & 56.94 & 39.95 & 14.85 & SmallDependenceEmphasis & 4.34 & 5.97 & 3.60 & 2.39 \\
\hline ClusterProminence & 16.89 & 75.29 & 20.99 & 19.57 & SmallDependenceHighGrayLevelEmphasis & 19.39 & 42.05 & 11.23 & 10.99 \\
\hline Imc1 & 6.10 & 8.35 & 7.34 & 3.16 & SmallDependenceLowGrayLevelEmphasis & 21.93 & 10.94 & 22.51 & 6.38 \\
\hline Imc2 & 0.22 & 0.57 & 0.65 & 0.41 & & & & & \\
\hline
\end{tabular}

\begin{tabular}{|c|c|c|c|c|}
\hline NGTDM & $\begin{array}{l}\text { A } \\
\text { (T1) }\end{array}$ & $\stackrel{\text { B }}{(T 1)}$ & $\begin{array}{l}\text { A } \\
\text { (T2) }\end{array}$ & $\stackrel{\text { B }}{(T 2)}$ \\
\hline Busyness & 15.81 & 28.10 & 12.41 & 8.34 \\
\hline Coarseness & 9.25 & 2.84 & 10.35 & 1.21 \\
\hline Complexity & 12.00 & 49.44 & 11.93 & 12.96 \\
\hline Contrast & 16.93 & 26.73 & 17.60 & 7.60 \\
\hline Strength & 11.37 & 30.21 & 14.18 & 8.08 \\
\hline
\end{tabular}

Table 3. Median CV (\%) of radiomic features estimate for effect A and B for both T1 and T2 maps. The median CV was calculated across subjects and bin widths/voxel sizes for effect A/B. 


\begin{tabular}{llllll} 
first order & T1 & T2 & GLRLM & T1 & T2 \\
\hline Mean & 123.38 & 137.01 & GrayLevelNonUniformity & 56.94 & 59.79 \\
\hline Variance & 157.96 & 162.00 & GrayLevelNonUniformityNormalized & 64.65 & 67.31 \\
\hline Skewness & 290.63 & 124.54 & GrayLevelVariance & 156.84 & 160.07 \\
\hline Kurtosis & 102.27 & 83.10 & HighGrayLevelRunEmphasis & 153.34 & 124.01 \\
\hline Median & 123.39 & 137.89 & LongRunEmphasis & 19.75 & 21.85 \\
\hline Minimum & 179.51 & 323.76 & LongRunHighGrayLevelEmphasis & 143.84 & 122.48 \\
\hline 10Percentile & 129.29 & 148.96 & LongRunLowGrayLevelEmphasis & 238.91 & 196.44 \\
\hline 90Percentile & 119.12 & 126.82 & LowGrayLevelRunEmphasis & 214.81 & 179.96 \\
\hline Maximum & 98.00 & 94.83 & RunEntropy & 15.69 & 15.61 \\
\hline InterquartileRange & 87.09 & 81.68 & RunLengthNonUniformity & 15.03 & 16.41 \\
\hline MeanAbsoluteDeviation & 78.07 & 86.65 & RunLengthNonUniformityNormalized & 9.62 & 10.82 \\
\hline RobustMeanAbsoluteDeviation & 88.39 & 84.91 & RunPercentage & 5.93 & 6.46 \\
\hline Energy & 147.94 & 161.71 & RunVariance & 74.19 & 92.94 \\
\hline RootMeanSquared & 115.92 & 121.92 & ShortRunEmphasis & 4.27 & 4.76 \\
\hline Entropy & 22.16 & 22.65 & ShortRunHighGrayLevelEmphasis & 155.70 & 123.67 \\
\hline Uniformity & 68.06 & 69.71 & ShortRunLowGrayLevelEmphasis & 207.87 & 175.45 \\
\hline
\end{tabular}

\begin{tabular}{|c|c|c|c|c|c|}
\hline GLCM & $\mathrm{T} 1$ & T2 & GLSZM & $\mathrm{T} 1$ & T2 \\
\hline MaximumProbability & 88.76 & 89.84 & GrayLevelNonUniformity & 32.44 & 34.44 \\
\hline JointAverage & 81.60 & 79.79 & GrayLevelNonUniformityNormalized & 56.05 & 60.46 \\
\hline SumSquares & 157.56 & 156.98 & GrayLevelVariance & 154.66 & 154.80 \\
\hline JointEntropy & 15.78 & 16.98 & HighGrayLevelZoneEmphasis & 153.12 & 122.66 \\
\hline DifferenceAverage & 60.44 & 64.92 & LargeAreaEmphasis & 98.70 & 105.73 \\
\hline DifferenceVariance & 127.76 & 132.98 & LargeAreaHighGrayLevelEmphasis & 143.67 & 120.75 \\
\hline DifferenceEntropy & 20.72 & 23.57 & LargeAreaLowGrayLevelEmphasis & 258.03 & 215.60 \\
\hline SumEntropy & 19.58 & 19.92 & LowGrayLevelZoneEmphasis & 202.41 & 170.09 \\
\hline JointEnergy & 103.58 & 101.07 & SizeZoneNonUniformity & 36.45 & 39.43 \\
\hline Contrast & 123.98 & 126.37 & SizeZoneNonUniformityNormalized & 19.03 & 22.57 \\
\hline Id & 27.61 & 36.75 & SmallAreaEmphasis & 9.36 & 11.92 \\
\hline Idn & 2.01 & 2.04 & SmallAreaHighGrayLevelEmphasis & 159.11 & 121.66 \\
\hline $\mathrm{Idm}$ & 38.71 & 52.74 & SmallAreaLowGrayLevelEmphasis & 173.29 & 152.38 \\
\hline Idmn & 0.45 & 0.47 & ZoneEntropy & 9.40 & 9.15 \\
\hline InverseVariance & 32.53 & 46.06 & ZonePercentage & 19.89 & 22.20 \\
\hline Correlation & 70.13 & 69.79 & ZoneVariance & 141.93 & 140.58 \\
\hline Autocorrelation & 153.87 & 122.41 & & & \\
\hline ClusterTendency & 164.02 & 171.18 & GLDM & $\mathrm{T} 1$ & T2 \\
\hline ClusterShade & 240.89 & 258.39 & DependenceEntropy & 10.03 & 9.67 \\
\hline ClusterProminence & 235.08 & 239.88 & DependenceNonUniformity & 31.40 & 33.09 \\
\hline Imc1 & 38.00 & 42.61 & DependenceNonUniformity Normalized & 31.40 & 33.09 \\
\hline \multirow[t]{2}{*}{ Imc2 } & 10.38 & 12.14 & DependenceVariance & 62.51 & 72.02 \\
\hline & & & $\begin{array}{l}\text { GrayLevelNonUniformity } \\
\text { Page } 8 / 18\end{array}$ & 68.06 & 69.71 \\
\hline
\end{tabular}




\begin{tabular}{llllll}
\hline NGTDM & T1 & T2 & GrayLevelVariance & 157.95 & 161.99 \\
\hline Busyness & 213.50 & 167.31 & HighGrayLevelEmphasis & 153.44 & 124.29 \\
\hline Coarseness & 25.32 & 16.66 & LargeDependenceEmphasis & 52.59 & 66.06 \\
\hline Complexity & 170.55 & 155.95 & LargeDependenceHighGrayLevelEmphasis & 131.67 & 121.00 \\
\hline Contrast & 105.02 & 122.49 & LargeDependenceLowGrayLevelEmphasis & 254.19 & 213.45 \\
\hline Strength & 138.12 & 138.02 & LowGrayLevelEmphasis & 218.28 & 185.60 \\
\hline & & & SmallDependenceEmphasis & 22.65 & 24.83 \\
\hline & & & SmallDependenceHighGrayLevelEmphasis & 170.28 & 122.94 \\
\hline & & & SmallDependenceLowGrayLevelEmphasis & 156.56 & 144.56
\end{tabular}

The results of the analysis of the linear correlation between radiomic features estimate and voxel size/bin width are reported in Supplementary Tables S1/S2 and Supplementary Tables S3/S4 for T1 and T2 maps, respectively. Most of the textural features showed a significant linear correlation with voxel size and bin width within the considered range of variation, with some exceptions (see Supplementary Tables S3) among radiomic features estimated from T2 maps when varying resampling voxel size (i.e., 6 GLCM, 7 GLRLM, 6 GLSZM, 6 GLDM, and 3 NGTDM radiomic features).

\section{Discussion}

Recently, some studies have applied radiomic analysis to CMR imaging in order to assess whether this tool could reveal myocardial phenotypic alterations in HCM patients ${ }^{18-21,31-36}$. For instance, Baeßler et al. ${ }^{31}$ have performed ML classification based on texture analysis of T1-weighted CMR images, and they have found, through logistic regression analysis, a model which discriminated between HCM patients and control subjects, with a sensitivity/specificity of $91 \% / 93 \%$. Alis et al. ${ }^{35}$ have reported that texture analysis of LGE images could classify HCM patients with and without ventricular tachyarrhythmia. They have evaluated the diagnostic performances of four ML-based methods (i.e., random forest, k-nearest neighbors, support vector machine, and naïve Bayes), reaching diagnostic accuracies (assessed by 10 -fold cross-validation) ranging from $82.3-94.1 \%$. Amano et al. ${ }^{18}$, using radiomic features from T2-weighted STIR images, have found that a GLRLM-GrayLevelNonUniformity value of 64.7 was the best discriminator between $\mathrm{HCM}$ patients and controls with an area under the receiver operating characteristic curve of 0.93 . Notably, only a few pilot $\mathrm{HCM}$ studies ${ }^{19-21,36}$ have applied radiomic analysis to T1 maps. Two studies ${ }^{19,21}$ have focused on the diagnostic capability of radiomic analysis to discriminate between HCM and HHD patients. In particular, Neisius et al. ${ }^{19}$, through a support vector machine classifier with 10 -fold cross-validation, have identified six texture features (two GLRLM features and four local binary pattern features) which yielded a diagnostic accuracy of $80 \%$ in classifying the two groups of patients. Shi et al. ${ }^{21}$, using two combinations of texture features estimated from T1 maps, have obtained (by means of logistic regression models with 8 fold cross-validation) an area under the receiver operating characteristic curve of 0.97 and of 0.80 in differentiating patients with HCM/HHD from healthy subjects and HCM from HHD patients, respectively. Moreover, the study by Neisius et al. ${ }^{36}$ has shown that radiomic analysis applied on T1 maps could be used as a potential decision support tool prior to gadolinium administration. Indeed, they have employed a decision tree ensemble with 10 -fold crossvalidation and identified five texture features (one GLCM feature, one GLRLM feature, and three local binary pattern features) which were able to distinguish between LGE + and LGE-, with an area under the receiver operating characteristic curve of 0.74 assessed in a testing cohort. Wang et al. ${ }^{20}$ have assessed the genotypic-phenotypic association in HCM patients through radiomic and ML analyses of T1 maps. They have reported no significant differences between two subgroups of patients (MYH7 and MYBPC3) when using conventional CMR imaging analyses (i.e., cardiac function, LGE, and native T1). Nonetheless, radiomic analysis of T1 maps reached a discrimination accuracy of $85.5 \%$ when employing a linear support vector machine classifier on a test validation dataset.

Previous studies have investigated the effect of image preprocessing on radiomic features estimation as a function of various factors, which include imaging technique/modality and anatomical region $40-43,47,48,51-55,72$. Nonetheless, to the best of our knowledge, so far, no previous study has assessed the effect of both image preprocessing and image filtering on radiomic features estimated from CMR T1 and T2 maps. Thus, in a group of HCM patients, we performed a rather comprehensive analysis, which considered multiple elements such as resampling voxel size, image discretization, and image filters.

In radiomic studies, voxel size resampling is a recommended and employed preprocessing step when analyzing data with different acquisition protocols or from different scanners, which can result in different acquisition voxel sizes ${ }^{59}$. For both T1 and T2 maps, we found that textural features belonging to GLCM and NGTDM classes showed moderate relative variability $(0.75<I C C \leq 0.9)$ when varying resampling voxel size, except for a limited number of radiomic features (Fig. 1). Conversely, the estimate of textural features of GLRLM, GLSZM, and GLDM classes showed a higher relative variability when varying resampling voxel size, with few exceptions (e.g., GrayLevelVariance from GLRLM, GLSZM, and GLDM, which presented ICC > 0.9 for both T1 and T2 maps). In general, radiomic features belonging to shape and first order classes were characterized by higher ICC values than textural features (Fig. 1), indicating that resampling voxel size has less impact on their estimate. In particular, shape radiomic features had ICC values $>0.9$ (Elongation was the only exception, showing $0.75<\mathrm{ICC} \leq 0.9$ ) and median CV values within $0.8 \%-2.7 \%$ (Table 2) for both T1 and T2 maps. Most of first order radiomic 
features presented ICC values $>0.75$, with only few features showing considerable (i.e., Energy from T1 and T2 maps, Kurtosis from T1 maps, and Skewness from T2 maps) or high (i.e., Kurtosis from T2 maps) relative variability. Furthermore, we revealed that even some radiomic features from T1 and T2 maps with ICC > 0.75 can have a non-negligible absolute variability in terms of CV (up to $30 \%$ or more, for single subjects) when varying resampling voxel size. In this regard, we found that T1 maps presented an appreciably higher number of radiomic features with ICC $>0.75$ and median CV $>15 \%$ than T2 maps (Figs. 4 and 5b). For several textural features, a significant linear correlation between their estimates and resampling voxel sizes was observed (Supplementary Tables S1 and S3).

Discretization of image intensity is another important step before radiomic features estimation, which allows simplifying rather complex computational operations. Given that T1 and T2 represent quantitative physical properties of tissues, we chose to apply a fixed bin width approach ${ }^{59}$. Bin width ranges of variation (i.e., 3.6-6.4 ms and $0.49-0.57 \mathrm{~ms}$ for T1 and T2 maps, respectively) were selected using the same criterion (i.e., median number of quantization levels between 30 and $130^{43,65,66}$ ), allowing a comparison between the results of T1 and T2 maps. A remarkable difference between T1 and T2 maps in sensitivity of radiomic features estimation to discretization was found. In general, textural features estimated from T1 maps showed higher variability than their estimates from T2 maps (ICC > 0.75). Only a limited number of T1 maps textural features were characterized by ICC $>0.75$ (Fig. 2 ) and some of them still had median CV > 15\% (Fig. 5a). Overall, CV values were greater for T1 maps (up to $75 \%$ ) than for T2 maps (less than $20 \%$ ) (see Table 2). As expected, shape and intensity-based statistical features (i.e., all first order features but Entropy and Uniformity) yielded ICC $=1$ and $C V=0 \%$. Indeed, these radiomic features are estimated by PyRadiomics, according to the IBSI recommendation, prior to discretization ${ }^{59}$ and hence this preprocessing step does not affect their estimate. The repeated measures correlation analysis showed that all radiomic textural features (with few exceptions) have a significant linear correlation between their estimates and bin width (Supplementary Tables S2 and S4).

Digital image filters can be applied before radiomic features extraction to detect and emphasize tissue characteristics different from those usually obtained from original (i.e., unfiltered) images. In this regard, the IBSI has proposed a new reference manual, in order to define and standardize the implementation of image filters in radiomics software ${ }^{60}$. We observed a relevant sensitivity of both first order and textural features estimates to image filter, with ICC $\leq 0.5$ (Fig. 3). This result, which was confirmed by the corresponding high CV values (Table 3 ), was not unexpected for first order radiomic features, given that image filters can strongly modify image intensity. However, the entity of this effect was not a priori predictable for textural radiomic features.

The revealed effect of resampling voxel size and bin width on the estimate of myocardial radiomic features from T1 and T2 mapping, albeit limited in many cases, sustains the importance of reporting/describing in detail these aspects in clinical and research studies. In this regard, while previous studies involving myocardial T1 mapping have described the radiomic features extraction process quite exhaustively ${ }^{19-21,36}$, some information about image preprocessing was still missing. For instance, none of these studies have reported whether and how image discretization was performed (notably, Neisius et al. ${ }^{19}$ have specified the number of discretized intensity levels but only for the radiomic features belonging to the GLRLM class, while no discretization was indicated for the GLCM class). Furthermore, the studies conducted by Neisius et al. ${ }^{19}$ and Shi et al. ${ }^{21}$ have acquired images with different in-plane spatial resolution (i.e., 2.1 and $1.3 \mathrm{~mm}$, respectively) and employed slightly different preprocessing steps (e.g., range re-segmentation and intensity outlier filtering, respectively ${ }^{59}$ ). This could partly explain the obtained different results in terms of discriminative radiomic features and accuracy.

So far, previous HCM radiomic studies ${ }^{19-21,36}$ have exploited only T1 mapping, mainly because of its capability of revealing myocardial fibrosis. However, T2 maps are considered the gold standard for the evaluation of myocardial edema, which is a well-known negative prognostic factor in $\mathrm{HCM}^{15,16}$. Moreover, although T2 mapping is not per se capable of evaluating myocardial fibrosis, texture analyses might theoretically overcome this limitation, unveiling myocardial structural heterogeneity due to myofibrillar disarray and fibrosis ${ }^{18,27}$. Therefore, given also that, overall, radiomic features from T2 maps have proven to be characterized by a lower sensitivity to image preprocessing than radiomic features from T1 maps (especially when varying bin width), radiomics from T2 mapping might be exploited to obtain a complementary characterization of HCM, albeit this lower sensitivity does not necessarily imply higher discriminative or predictive power. In addition, it should be noted that, for both T1 and T2 mapping, the sensitivity of several radiomic features to resampling voxel size and bin width resulted rather independent of bin width and resampling voxel size, respectively. Moreover, only a limited number of radiomic features showed high relative variability $(\mathrm{ICC} \leq 0.5)$ associated with different resampling voxel size or bin width. Some radiomic features with ICC $>0.75$ still presented high variability $(>30 \%)$ in terms of CV for single subjects, suggesting that such radiomic data from different centers should be compared or pooled with caution.

\section{Conclusions}

Toward an optimization and standardization in radiomic applications, any potential dependence of radiomic features on various aspect of the radiomic workflow should be possibly taken into account when planning a clinical or research study. Nowadays, there is a growing interest in exploiting radiomic techniques in various clinical applications. In particular, the study of myocardial diseases represents an emerging and promising field of application. In this HCM study, while we observed a sensitivity of myocardial radiomic features from T1 and T2 mapping to image preprocessing, this effect was very remarkable only when considering image filtering. The sensitivity of radiomic features to different resampling voxel sizes and bin widths was limited for several textural radiomic features, as well as for shape and first order radiomic features. The estimate of several textural radiomic features showed a linear significant correlation with resampling voxel size and bin width. In general, radiomic features from T1 mapping were more sensitive to image preprocessing than radiomic features from T2 mapping, especially when varying bin width. Overall, our results might corroborate the potential of radiomics from $\mathrm{T} 1$ and $\mathrm{T} 2$ mapping in $\mathrm{HCM}$ and hopefully in other myocardial diseases. 


\section{Declarations}

\section{AUTHOR CONTRIBUTIONS}

CT, DM, and MG conceived the original idea and drafted the manuscript. Magnetic resonance imaging acquisitions were carried out by CT, JDM, and LS. Image and statistical analyses were performed by DM. DM and MG prepared all figures and tables. All authors contributed to the interpretation of the results and revised the final version of the manuscript.

\section{DATA AVAILABILITY}

The datasets generated during and/or analysed during the current study are available from the corresponding author on reasonable request.

\section{COMPETING INTERESTS}

All authors declare no competing interests.

\section{References}

1. Baxi, A. J. et al. Hypertrophic cardiomyopathy from A to Z: genetics, pathophysiology, imaging, and management. RadioGraphics $36,335-354$ (2016).

2. ESC guidelines on diagnosis and management of hypertrophic cardiomyopathy: the task force for the diagnosis and management of hypertrophic cardiomyopathy of the European Society of Cardiology (ESC). Eur Heart J 35, 2733-2779 (2014).

3. Rowin, E. J., Maron, B. J. \& Maron, M. S. The hypertrophic cardiomyopathy phenotype viewed through the prism of multimodality imaging. JACC: Cardiovascular Imaging 13, 2002-2016 (2020).

4. Baggiano, A. et al. Role of CMR mapping techniques in cardiac hypertrophic phenotype. Diagnostics 10, 770 (2020).

5. Dass, S. et al. Myocardial tissue characterization using magnetic resonance noncontrast T1 mapping in hypertrophic and dilated cardiomyopathy. Circ Cardiovasc Imaging 5, 726-733 (2012).

6. Kato, S. et al. Myocardial native T1 time in patients with hypertrophic cardiomyopathy. The American Journal of Cardiology 118, 1057-1062 (2016).

7. Huang, L. et al. MRI native T1 and T2 mapping of myocardial segments in hypertrophic cardiomyopathy: tissue remodeling manifested prior to structure changes. BJR 92, 20190634 (2019).

8. McLellan, A. J. A. et al. Diffuse ventricular fibrosis on cardiac magnetic resonance imaging associates with ventricular tachycardia in patients with hypertrophic cardiomyopathy. J Cardiovasc Electrophysio/ 27, 571-580 (2016).

9. Sado, D. M. et al. Cardiovascular magnetic resonance measurement of myocardial extracellular volume in health and disease. Heart 98 , $1436-1441$ (2012)

10. Swoboda, P. P. et al. Assessing myocardial extracellular volume by T1 mapping to distinguish hypertrophic cardiomyopathy from athlete's heart. Journal of the American College of Cardiology 67, 2189-2190 (2016).

11. Avanesov, M. et al. Prediction of the estimated 5-year risk of sudden cardiac death and syncope or non-sustained ventricular tachycardia in patients with hypertrophic cardiomyopathy using late gadolinium enhancement and extracellular volume CMR. Eur Radio/ 27, 5136-5145 (2017).

12. Haaf, P. et al. Cardiac T1 mapping and extracellular volume (ECV) in clinical practice: a comprehensive review. J Cardiovasc Magn Reson 18,89 (2017)

13. Kuusisto, J. et al. Low-grade inflammation and the phenotypic expression of myocardial fibrosis in hypertrophic cardiomyopathy. Heart 98 , 10071013 (2012).

14. Kolman, L. et al. Abnormal lymphatic channels detected by T2-weighted MR imaging as a substrate for ventricular arrhythmia in HCM. JACC: Cardiovascular Imaging 9, 1354-1356 (2016).

15. Amano, Y., Aita, K., Yamada, F., Kitamura, M. \& Kumita, S. Distribution and clinical significance of high signal intensity of the myocardium on T2weighted images in 2 phenotypes of hypertrophic cardiomyopathy. Journal of Computer Assisted Tomography 39, 951-955 (2015).

16. Todiere, G. et al. Abnormal T2-STIR magnetic resonance in hypertrophic cardiomyopathy: a marker of advanced disease and electrical myocardial instability. PLOS ONE 9, e111366 (2014).

17. Amano, Y. et al. Myocardial T2 mapping in patients with hypertrophic cardiomyopathy. Journal of Computer Assisted Tomography 41, 344-348 (2017).

18. Amano, Y. et al. Detection of myocardial tissue alterations in hypertrophic cardiomyopathy using texture analysis of T2-weighted short inversion time inversion recovery magnetic resonance imaging. Journal of Computer Assisted Tomography 44, 341-345 (2020).

19. Neisius, U. et al. Radiomic analysis of myocardial native T1 imaging discriminates between hypertensive heart disease and hypertrophic cardiomyopathy. JACC: Cardiovascular Imaging 12, 1946-1954 (2019).

20. Wang, J. et al. Radiomic analysis of native T1 mapping images discriminates between MYH7 and MYBPC3-related hypertrophic cardiomyopathy. $J$ Magn Reson Imaging 52, 1714-1721 (2020). 
21. Shi, R.-Y. et al. Texture analysis applied in T1 maps and extracellular volume obtained using cardiac MRI in the diagnosis of hypertrophic cardiomyopathy and hypertensive heart disease compared with normal controls. Clinical Radiology 76, 236.e9-236.e19 (2021).

22. Gillies, R. J., Kinahan, P. E. \& Hricak, H. Radiomics: images are more than pictures, they are data. Radiology 278, 563-577 (2016).

23. Lambin, P. et al. Radiomics: the bridge between medical imaging and personalized medicine. Nat Rev Clin Onco/ 14, 749-762 (2017).

24. van Timmeren, J. E., Cester, D., Tanadini-Lang, S., Alkadhi, H. \& Baessler, B. Radiomics in medical imaging-“how-to" guide and critical reflection. Insights Imaging 11, 91 (2020).

25. Raisi-Estabragh, Z. et al. Cardiac magnetic resonance radiomics: basic principles and clinical perspectives. European Heart Journal - Cardiovascular Imaging 21, 349-356 (2020).

26. Hassani, C., Saremi, F., Varghese, B. A. \& Duddalwar, V. Myocardial radiomics in cardiac MRI. American Journal of Roentgenology 214, 536-545 (2020)

27. Amano, Y. et al. Clinical importance of myocardial $\mathrm{T}_{2}$ mapping and texture analysis. MRMS 20, 139-151 (2021).

28. Baessler, B. et al. Subacute and chronic left ventricular myocardial scar: accuracy of texture analysis on nonenhanced cine MR images. Radiology 286, 103-112 (2018).

29. Baessler, B. et al. Cardiac MRI texture analysis of T1 and T2 maps in patients with infarctlike acute myocarditis. Radiology 289, 357-365 (2018).

30. Baessler, B. et al. Cardiac MRI and texture analysis of myocardial T1 and T2 maps in myocarditis with acute versus chronic symptoms of heart failure. Radiology 292, 608-617 (2019).

31. Baeßler, B., Mannil, M., Maintz, D., Alkadhi, H. \& Manka, R. Texture analysis and machine learning of non-contrast T1-weighted MR images in patients with hypertrophic cardiomyopathy - preliminary results. European Journal of Radiology 102, 61-67 (2018).

32. Schofield, R. et al. Texture analysis of cardiovascular magnetic resonance cine images differentiates aetiologies of left ventricular hypertrophy. Clinical Radiology 74, 140-149 (2019).

33. Amano, Y., Suzuki, Y., Yanagisawa, F., Omori, Y. \& Matsumoto, N. Relationship between extension or texture features of late gadolinium enhancement and ventricular tachyarrhythmias in hypertrophic cardiomyopathy. BioMed Research International 2018, 1-6 (2018).

34. Cheng, S. et al. LGE-CMR-derived texture features reflect poor prognosis in hypertrophic cardiomyopathy patients with systolic dysfunction: preliminary results. Eur Radio/ 28, 4615-4624 (2018).

35. Alis, D., Guler, A., Yergin, M. \& Asmakutlu, O. Assessment of ventricular tachyarrhythmia in patients with hypertrophic cardiomyopathy with machine learning-based texture analysis of late gadolinium enhancement cardiac MRI. Diagnostic and Interventional Imaging 101, 137-146 (2020).

36. Neisius, U. et al. Texture signatures of native myocardial $T_{1}$ as novel imaging markers for identification of hypertrophic cardiomyopathy patients without scar. J Magn Reson Imaging 52, 906-919 (2020).

37. Cattell, R., Chen, S. \& Huang, C. Robustness of radiomic features in magnetic resonance imaging: review and a phantom study. Vis. Comput. Ind. Biomed. Art 2, 19 (2019).

38. Traverso, A., Wee, L., Dekker, A. \& Gillies, R. Repeatability and reproducibility of radiomic features: a systematic review. International Journal of Radiation Oncology Biology Physics 102, 1143-1158 (2018).

39. Zhao, B. et al. Reproducibility of radiomics for deciphering tumor phenotype with imaging. Sci Rep 6, 23428 (2016).

40. Shafiq-ul-Hassan, M. et al. Intrinsic dependencies of CT radiomic features on voxel size and number of gray levels. Med. Phys. 44, 1050-1062 (2017).

41. Shafiq-ul-Hassan, M. et al. Voxel size and gray level normalization of CT radiomic features in lung cancer. Sci Rep 8, 10545 (2018).

42. Zwanenburg, A. Radiomics in nuclear medicine: robustness, reproducibility, standardization, and how to avoid data analysis traps and replication crisis. Eur J Nucl Med Mol Imaging 46, 2638-2655 (2019).

43. Leijenaar, R. T. H. et al. The effect of SUV discretization in quantitative FDG-PET radiomics: the need for standardized methodology in tumor texture analysis. Sci Rep 5, 11075 (2015).

44. Baeßler, B., Weiss, K. \& Pinto dos Santos, D. Robustness and reproducibility of radiomics in magnetic resonance imaging: a phantom study. Investigative Radiology 54, 221-228 (2019).

45. Fiset, S. et al. Repeatability and reproducibility of MRI-based radiomic features in cervical cancer. Radiotherapy and Oncology 135, 107-114 (2019).

46. Peerlings, J. et al. Stability of radiomics features in apparent diffusion coefficient maps from a multi-centre test-retest trial. Sci Rep 9, 4800 (2019).

47. Schwier, M. et al. Repeatability of multiparametric prostate MRI radiomics features. Sci Rep 9, 9441 (2019).

48. Shiri, I. et al. Repeatability of radiomic features in magnetic resonance imaging of glioblastoma: test-retest and image registration analyses. Med. Phys. 47, 4265-4280 (2020).

49. Lecler, A. et al. Combining multiple magnetic resonance imaging sequences provides independent reproducible radiomics features. Sci Rep 9 , 2068 (2019).

50. Duron, L. et al. Gray-level discretization impacts reproducible MRI radiomics texture features. PLoS ONE 14, e0213459 (2019).

51. Traverso, A. et al. Stability of radiomic features of apparent diffusion coefficient (ADC) maps for locally advanced rectal cancer in response to image pre-processing. Physica Medica 61, 44-51 (2019). 
52. Traverso, A. et al. Sensitivity of radiomic features to inter-observer variability and image pre-processing in Apparent Diffusion Coefficient (ADC) maps of cervix cancer patients. Radiotherapy and Oncology 143, 88-94 (2020).

53. Molina, D. et al. Lack of robustness of textural measures obtained from 3D brain tumor MRIs impose a need for standardization. PLoS ONE 12, e0178843 (2017).

54. Brynolfsson, P. et al. Haralick texture features from apparent diffusion coefficient (ADC) MRI images depend on imaging and pre-processing parameters. Sci Rep 7, 4041 (2017).

55. Park, S.-H. et al. Robustness of magnetic resonance radiomic features to pixel size resampling and interpolation in patients with cervical cancer. Cancer Imaging 21, 19 (2021).

56. Jang, J. et al. Reproducibility of segmentation-based myocardial radiomic features with cardiac MRI. Radiology: Cardiothoracic Imaging 2, e190216 (2020).

57. Jang, J. et al. Sensitivity of Myocardial Radiomic Features to Imaging Parameters in Cardiac MR Imaging. J Magn Reson Imaging jmri.27581 (2021) doi:10.1002/jmri.27581.

58. Alis, D., Yergin, M., Asmakutlu, O., Topel, C. \& Karaarslan, E. The influence of cardiac motion on radiomics features: radiomics features of nonenhanced CMR cine images greatly vary through the cardiac cycle. Eur Radio/31, 2706-2715 (2021).

59. Zwanenburg, A., Leger, S., Vallières, M. \& Löck, S. Image biomarker standardisation initiative. (2016).

60. Depeursinge, A. et al. Standardised convolutional filtering for radiomics. arXiv:2006.05470 (2021).

61. Messroghli, D. R. et al. Human myocardium: single-breath-hold MR T1 mapping with high spatial resolution - reproducibility study. Radiology 238, 1004-1012 (2006).

62. Giri, S. et al. T2 quantification for improved detection of myocardial edema. J Cardiovasc Magn Reson 11, 56 (2009).

63. Kikinis, R., Pieper, S. D. \& Vosburgh, K. G. 3D Slicer: a platform for subject-specific image analysis, visualization, and clinical support. in Intraoperative Imaging and Image-Guided Therapy (ed. Jolesz, F. A.) 277-289 (Springer New York, 2014). doi:10.1007/978-1-4614-7657-3_19.

64. Fedorov, A. et al. 3D Slicer as an image computing platform for the quantitative imaging network. Magnetic Resonance Imaging 30, $1323-1341$ (2012)

65. Yip, S. S. F. \& Aerts, H. J. W. L. Applications and limitations of radiomics. Phys. Med. Biol. 61, R150-R166 (2016).

66. Tixier, F. et al. Intratumor heterogeneity characterized by textural features on baseline 18F-FDG PET images predicts response to concomitant radiochemotherapy in esophageal cancer. Journal of Nuclear Medicine 52, 369-378 (2011).

67. van Griethuysen, J. J. M. et al. Computational radiomics system to decode the radiographic phenotype. Cancer Res 77, e104-e107 (2017).

68. Shrout, P. E. \& Fleiss, J. L. Intraclass correlations: uses in assessing rater reliability. Psychological Bulletin 86, 420-428 (1979).

69. McGraw, K. O. \& Wong, S. P. Forming inferences about some intraclass correlation coefficients. Psychological Methods 1, 30-46 (1996).

70. Bakdash, J. Z. \& Marusich, L. R. Repeated measures correlation. Front. Psychol. 8, 456 (2017).

71. R Core Team; R: a language and environment for statistical computing. https://www.R-project.org/ (2019).

72. Fave, X. et al. Impact of image preprocessing on the volume dependence and prognostic potential of radiomics features in non-small cell lung cancer. Transl. Cancer Res. 5, 349-363 (2016).

\section{Figures}




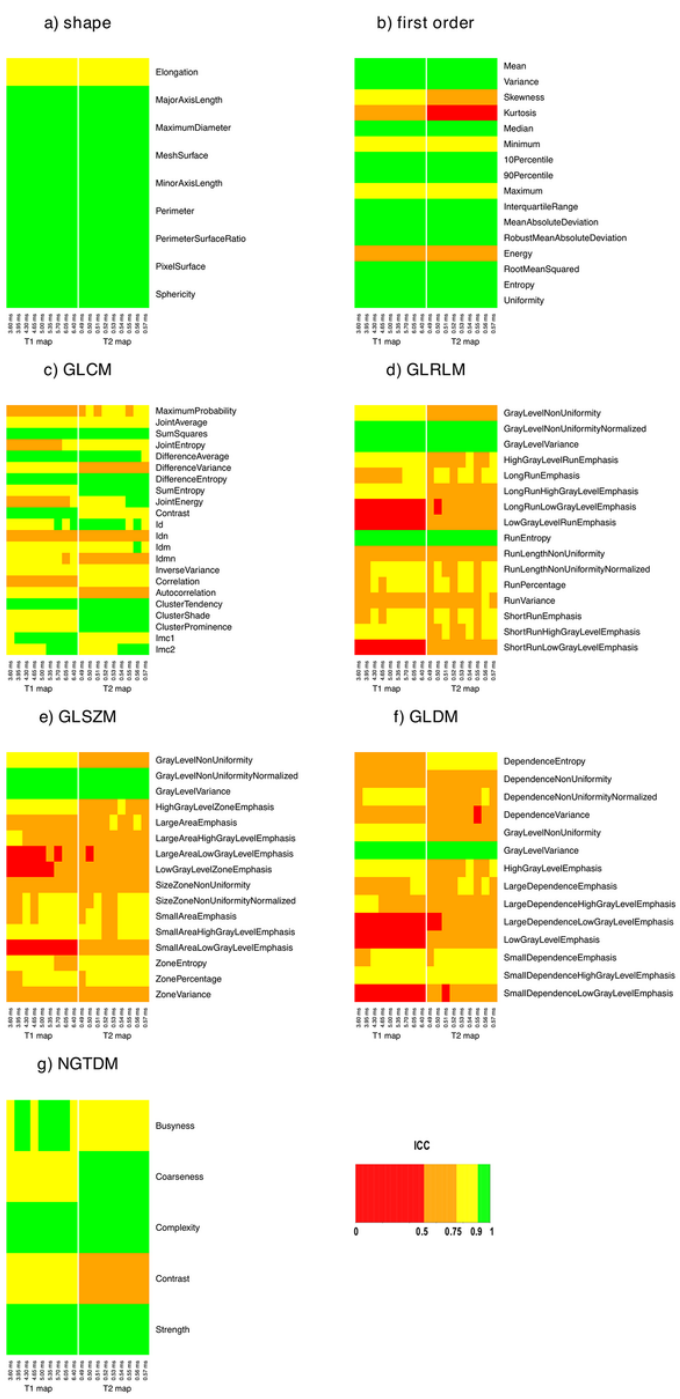

\section{Figure 1}

ICC results for effect A for both T1 and T2 maps. The heatmap of each radiomic features class (i.e., shape, first order, GLCM, GLRLM, GLSZM, GLDM, and NGTDM) shows the degree of relative variability in radiomic features estimate when using different resampling voxel sizes for different bin widths (i.e., $3.60,3.95,4.30,4.65,5.00,5.35,5.70,6.05$, and 6.40 ms for T1 maps and $0.49,0.50,0.51,0.52,0.53,0.54,0.55,0.56$, and 0.57 ms for T2 maps). 

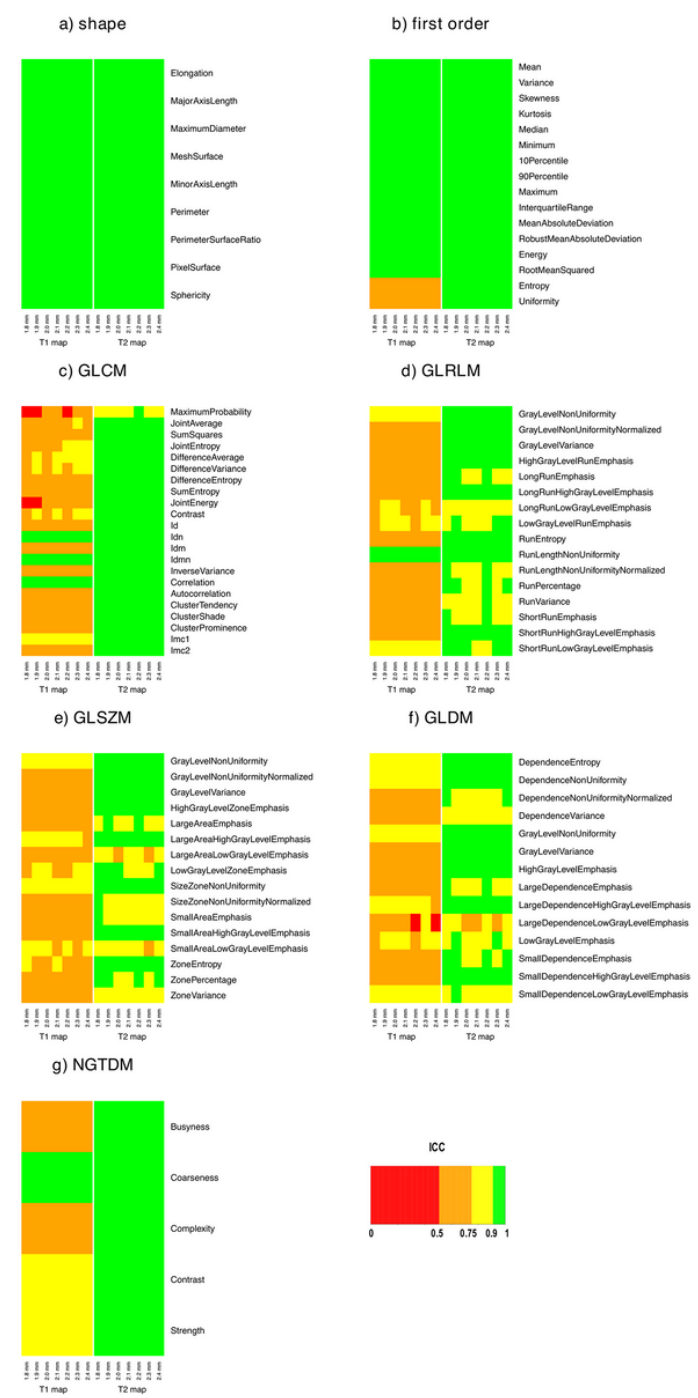

\section{Figure 2}

ICC results for effect B for both T1 and T2 maps. The heatmap of each radiomic features class (i.e., shape, first order, GLCM, GLRLM, GLSZM, GLDM, and NGTDM) shows the degree of relative variability in radiomic features estimate when using different bin widths for different resampling voxel sizes (i.e., $1.8,1.9,2.0,2.1,2.2,2.3$, and $2.4 \mathrm{~mm}$ for both $\mathrm{T} 1$ and $\mathrm{T} 2 \mathrm{maps})$. 
a) first order

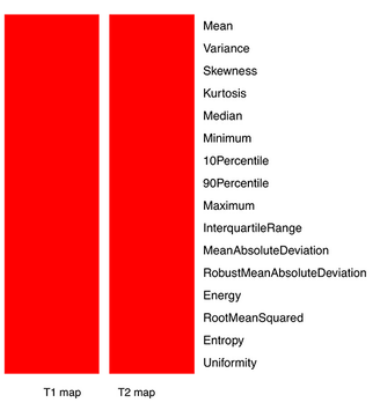

d) GLRLM

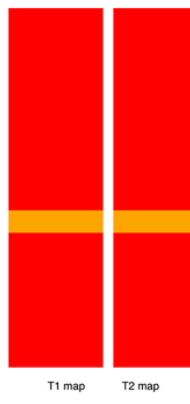

f) GLDM
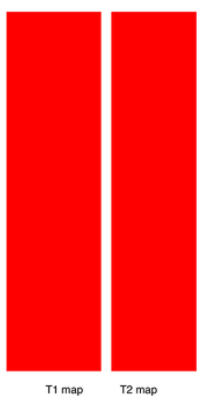

c) GLCM

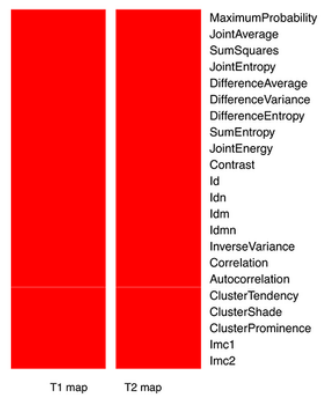

e) GLSZM

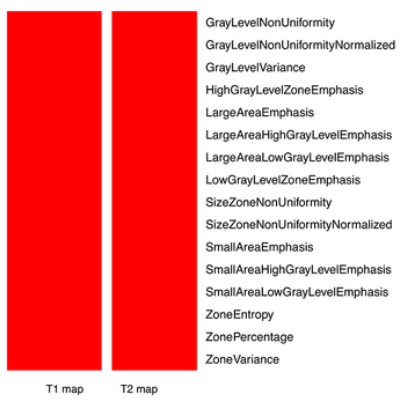

g) NGTDM
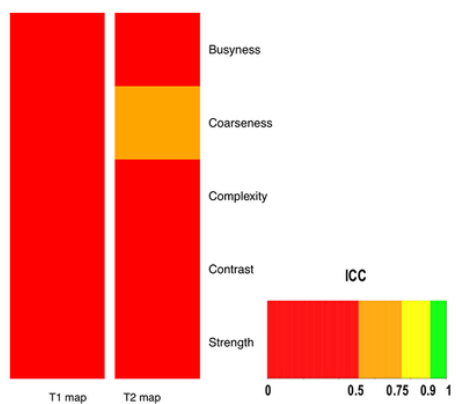

Figure 3

ICC results for effect $\mathrm{C}$ for both T1 and T2 maps. The heatmap of each radiomic features class (i.e., first order, GLCM, GLRLM, GLSZM, GLDM, and NGTDM) shows the degree of relative variability in radiomic features estimate when using different spatial filters with fixed bin width (i.e., 6 ms and 0.56 ms for T1 and T2 maps, respectively) and resampling voxel size (i.e., $2.1 \mathrm{~mm}$ for both T1 and T2 maps). 

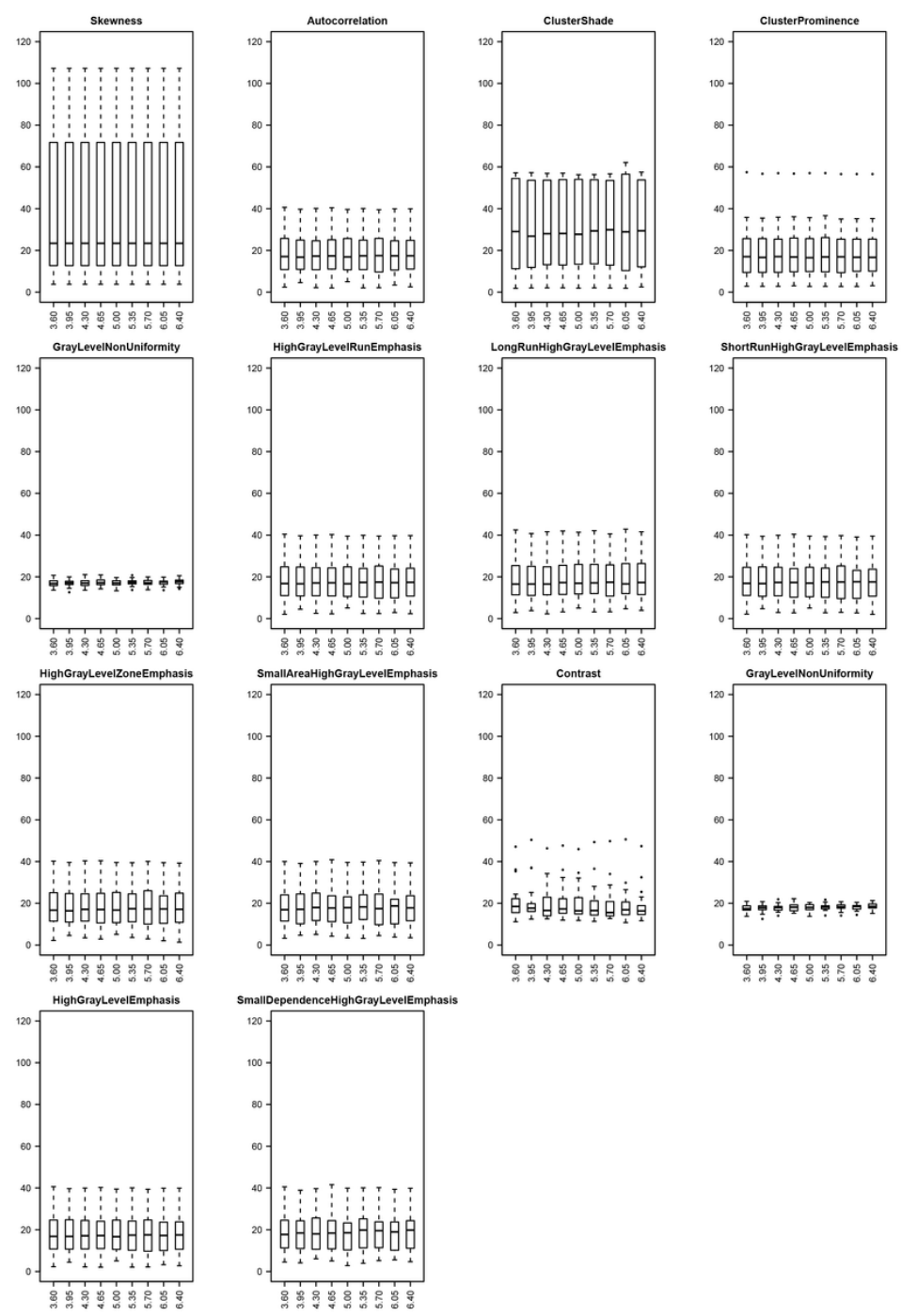

\section{Figure 4}

Box and whisker plots of CV (\%) values of enrolled subjects for effect A assessed on T1 maps. Radiomic features with both median (across subjects) CV $>15 \%$ and ICC > 0.75 for each bin width (i.e., 3.60, 3.95, 4.30, 4.65, 5.00, 5.35, 5.70, 6.05, and $6.40 \mathrm{~ms}$ ) are shown. 

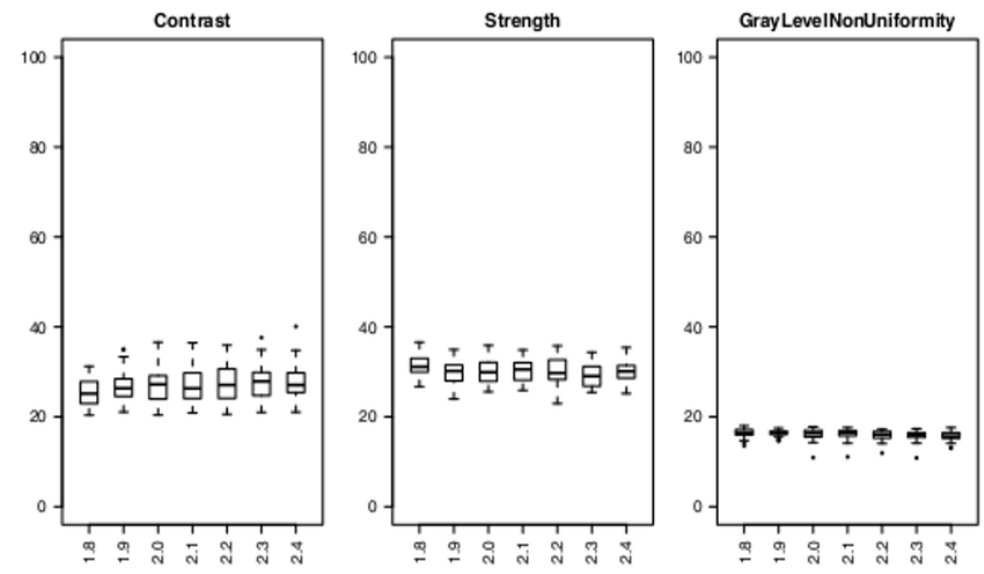

(a)

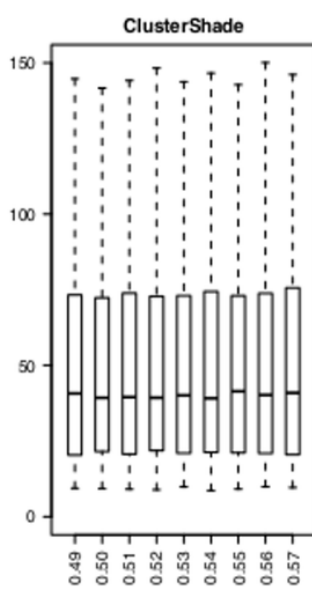

(b)

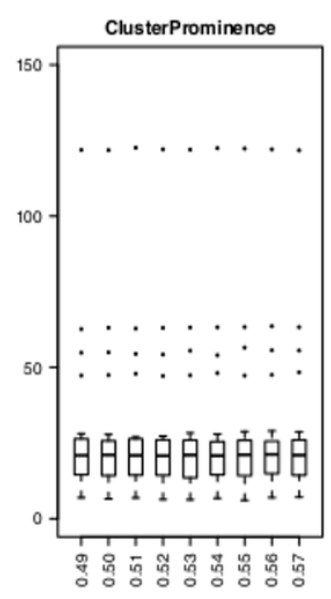

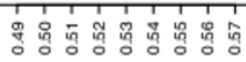

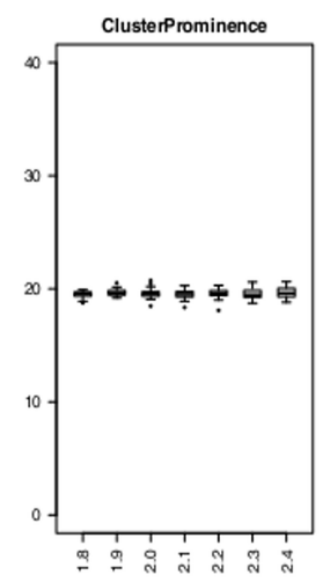

(c)

\section{Figure 5}

Box and whisker plots of CV (\%) values of enrolled subjects for effect B assessed on T1 maps (a) and for effect A (b)/B (c) assessed on T2 maps. (a, c) Radiomic features with both median (across subjects) CV > 15\% and ICC > 0.75 for each resampling voxel size (i.e., 1.8, 1.9, 2.0, 2.1, 2.2, 2.3, and 2.4) are shown for T1 (a) and T2 maps (c). (b) Radiomic features computed from T2 maps with both median (across subjects) CV > $15 \%$ and ICC > 0.75 for each bin width (i.e., $0.49,0.50,0.51,0.52,0.53,0.54,0.55,0.56$, and $0.57 \mathrm{~ms}$ ) are shown.

\section{Supplementary Files}

This is a list of supplementary files associated with this preprint. Click to download.

- SupplementaryMaterial.pdf 